\title{
Reliability Analysis of III-V Solar Cells Grown on Recycled GaAs Substrates and an Electroplated Nickel Substrate
}

\author{
Ray-Hua Horng, ${ }^{1}$ Ming-Chun Tseng, ${ }^{1}$ and Shui-Yang Lien ${ }^{2}$ \\ ${ }^{1}$ Graduate Institute of Precision Engineering, National Chung Hsing University, Taichung 402, Taiwan \\ ${ }^{2}$ Department of Materials Science and Engineering, DaYeh University, Changhua 515, Taiwan \\ Correspondence should be addressed to Ming-Chun Tseng; tseng1026@dragon.nchu.edu.tw
}

Received 14 June 2013; Revised 30 October 2013; Accepted 4 November 2013

Academic Editor: Antonino Bartolotta

Copyright (c) 2013 Ray-Hua Horng et al. This is an open access article distributed under the Creative Commons Attribution License, which permits unrestricted use, distribution, and reproduction in any medium, provided the original work is properly cited.

\begin{abstract}
This study involved analyzing the reliability of two types of III-V solar cells: (1) III-V solar cells grown on new and recycled gallium arsenide (GaAs) substrates and (2) the III-V solar cells transferred onto an electroplated nickel (Ni) substrate as III-V thin-film solar cells by using a cross-shaped pattern epitaxial lift-off (CPELO) process. The III-V solar cells were grown on new and recycled GaAs substrates to evaluate the reliability of the substrate. The recycled GaAs substrate was fabricated by using the CPELO process. The performance of the solar cells grown on the recycled GaAs substrate was affected by the uneven surface morphology of the recycled GaAs substrate, which caused the propagation of these dislocations into the subsequently grown active layer of the solar cell. The III-V solar cells were transferred onto an electroplated Ni substrate, which was also fabricated by using CPELO technology. The degradation of the III-V thin-film solar cell after conducting a thermal shock test could have been caused by microcracks or microvoids in the active layer or interface of the heterojunction, which resulted in the reduction of the external quantum efficiency response and the increase of recombination loss.
\end{abstract}

\section{Introduction}

High manufacturing cost is the major limitation for III-V electronic devices because the single crystal substrates (gallium arsenide, GaAs, or germanium, Ge, substrates) used to obtain the desired high crystalline quality are expensive. For example, multijunction III-V solar cells are always grown on single crystal GaAs substrates to prevent the degradation of the high bandgap junction caused by misfit dislocation from the low bandgap junction [1].

In general, single crystal substrates do not involve the photovoltaic effect. Therefore, the epitaxial structure of III-V solar cells can be transferred to any desired functional substrate [2]. Lightweight substrates [3] and materials with high thermal conductivities [4] can also be used as carrier substrates in space and terrestrial concentrator applications, respectively. Aluminum arsenide (AlAs) layers are usually used as the sacrificial layer between GaAs substrates and solar cell structures; therefore, epitaxial lift-off (ELO), a recycled substrate method, could potentially lower manufacturing costs. The chemical etchant used for the ELO process is an aqueous HF solution. However, prolonged exposure to the HF etchant increases the surface roughness of a wafer because of the byproduct from the chemical reaction between AlAs and the HF etchant [5], preventing the direct reuse of wafer and deteriorating the performance of a solar cell. To solve this problem, using different concentrations of chemical etchant to modify surface morphology of wafer was proposed [6]. Chemical cleaning is difficult to conduct because the etching mechanisms are sensitive to wafer contamination. Furthermore, the IBM Corporation [5] proposed using a surface-tension-assisted epitaxial lift-off (STA-ELO) process to separate a device layer from a GaAs substrate by using aqueous hydrochloric acid $(\mathrm{HCl})$, which selectively etches an AlInP sacrificial layer inserted between the device film and the substrate. The novel ELO process minimizes the amount of postetching residue and keeps the surface smooth, which leads to the direct reuse of the GaAs substrate. 


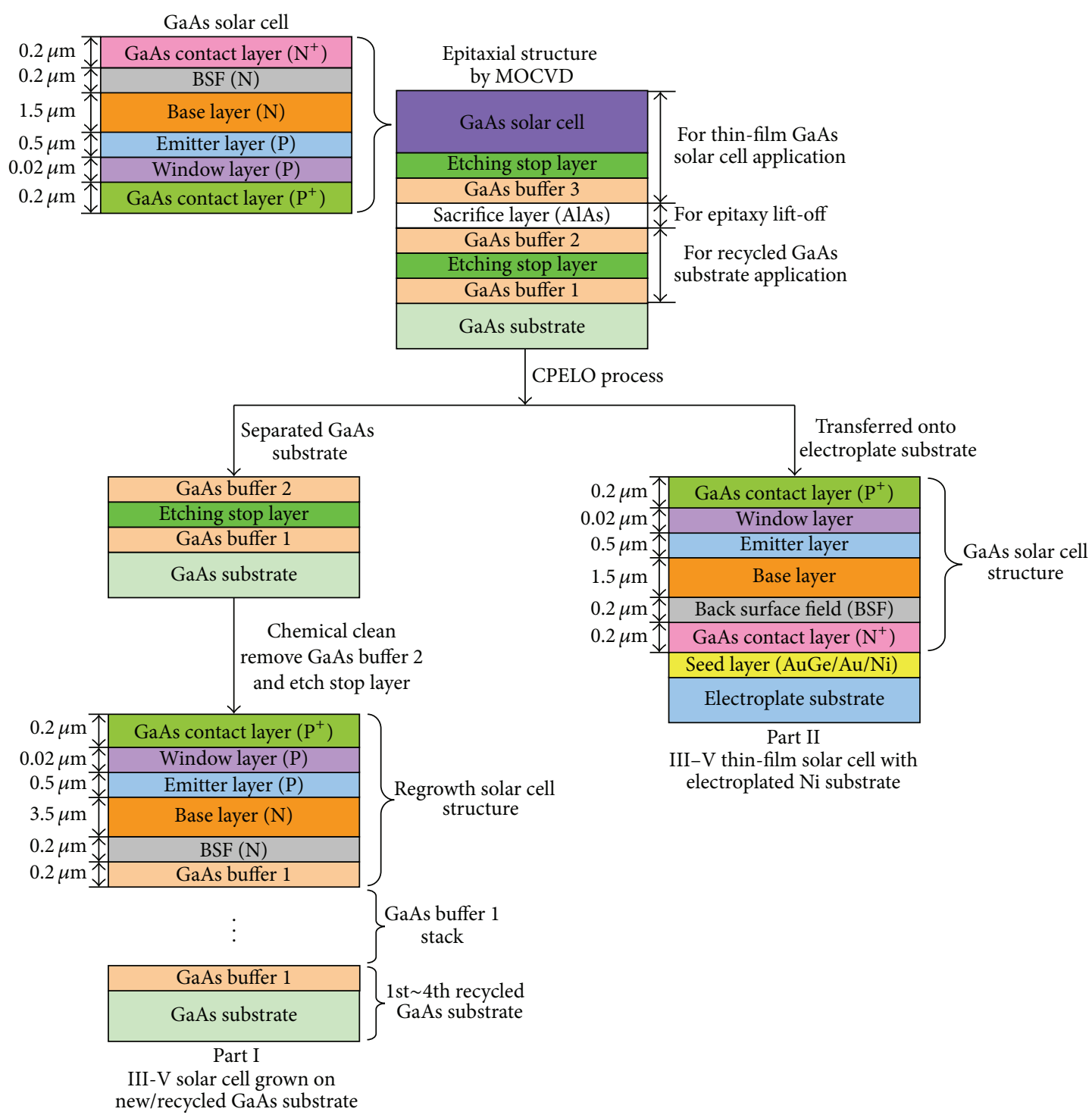

FIGURE 1: Device fabrication flowchart of experiment design.

In our previous study [7], an etching stop layer was added to the epitaxial structure of a III-V solar cell to prevent damage to the GaAs substrate caused by prolonged exposure to the HF solution. In addition, the etching stop layer protected the device structure of thin-film III-V solar cells during the lift-off process. Exposing the GaAs substrate to the HF solution roughens the surface of the GaAs substrate, which could be avoided by using a special epilayer design: GaAs substrate/GaAs buffer 1/etching stop layer/GaAs buffer 2/sacrificial layer/GaAs buffer 3/etch stop layer/device structure. Optimally, the GaAs substrate can be reused twice [7]. Applying the proposed technique substantially reduces the manufacturing cost. In this study, the performance reliability of an III-V solar cell grown on new and recycled GaAs substrates and III-V solar cells transferred onto an electroplated nickel (Ni) substrate as III-V thin-film solar cells were studied.

\section{Experiments}

Single junction GaAs solar cells were epitaxially grown by using low-pressure metalorganic chemical vapor deposition on 2-inch-diameter (100) GaAs wafers with a misorientation of $15^{\circ}$ toward $\langle 111\rangle$. The growth temperature and reactor pressure were $650^{\circ} \mathrm{C}$ and $60 \mathrm{mbar}$, respectively. The device fabrication flowchart is shown in Figure 1. The epitaxial structure of the GaAs solar cell consisted of GaAs buffer 1/etching stop layer/GaAs buffer 2/sacrifice layer (AlAs)/GaAs buffer 3/etching stop layer/heavily Mgdoped GaAs contact layer $(0.2 \mu \mathrm{m}) / \mathrm{Ga}_{0.49} \operatorname{In}_{0.51} \mathrm{P}: \mathrm{Mg}$ window layer $(0.02 \mu \mathrm{m}) / \mathrm{GaAs}: \mathrm{Mg}$ emitter layer $(0.5 \mu \mathrm{m}) / \mathrm{GaAs}: \mathrm{Si}$ base layer $(1.5 \mu \mathrm{m}) / \mathrm{Ga}_{0.49} \operatorname{In}_{0.51} \mathrm{P}: \mathrm{Si}$ back surface field (BSF; $0.2 \mu \mathrm{m}) /$ heavily Si-doped GaAs contact layer $(0.2 \mu \mathrm{m})$ (top of Figure 1). The GaAs solar cell structure was transferred onto 
an electroplated Ni substrate with a mirror structure as III-V thin-film solar cell by using a cross-shaped pattern epitaxial lift-off (CPELO) process, which is denoted as the "TF cell" and shown in the bottom right of Figure 1.

After the CPELO process was complete, the GaAs buffer 3 and etching stop layer were removed using a chemical solution. $\mathrm{AuGe} / \mathrm{Au} / \mathrm{Ni}(50 / 150 / 300 \mathrm{~nm})$ metal films were used to define the cross-shaped pattern array using photolithography. A cross-shaped hole was etched by using inductively coupled plasma-reactive ion etching (ICP-RIE) from the heavily Sidoped GaAs contact layer $\left(\mathrm{N}^{+}\right)$to GaAs buffer 2. The metal layer $(\mathrm{AuGe} / \mathrm{Au} / \mathrm{Ni})$ provided a hard mask for the ICPRIE dry etching process as well as a seed layer for the subsequently used $\mathrm{Ni}$ substrates ( $50 \mu \mathrm{m}$ thick), which were directly electroplated onto the bottom of the solar cell without using an adhesive layer. The bottom contact metal of the $\mathrm{AuGe} / \mathrm{Au} / \mathrm{Ni}$ layer was deposited onto the heavily Si-doped GaAs contact layer $\left(\mathrm{N}^{+}\right)$by using a thermal evaporation system. AuGe/Au/Ni functioned as an ohmic contact with the solar cell and a metal reflector for incident light. Details on the CPELO process, recycled GaAs substrate process, and solar cell fabrication process are described in the authors' previous study [7]. In this study, a conventional single junction solar cell was grown on the recycled GaAs substrate to evaluate the quality of this substrate, as shown in the bottom left of Figure 1. The regrowth and chemical cleaning process was repeated 1-4 times after performing the ELO process to form a recycled GaAs substrate. The solar cell fabricated on the new GaAs substrate was prepared and labeled "Initial" for comparison. The solar cell structures grown on the GaAs substrate were labeled "1st," "2nd," "3rd," and "4th" according to the number of times they were recycled for comparison. The solar cell fabrication procedure is described as follows. First the front grid metal of AuBe/Au with coverage of approximately $10 \%$ was deposited and annealed. After that, the bottom contact metal of $\mathrm{AuGe} / \mathrm{Au}$ was deposited on the heavily Si-doped GaAs layer by using thermal evaporation. Finally, $\mathrm{SiO}_{2}(n=1.42)$ and $\mathrm{TiO}_{2}(n=2.32)$ were deposited on the top surface as an antireflection coating to reduce the surface reflection of the solar cell. The external quantum efficiency (EQE) was measured by using the Newport-Oriel IQE-200 system with a quartz-tungsten halogen lamp and monochromator at $25^{\circ} \mathrm{C}$. The spot size was $1 \mathrm{~mm} \times 1 \mathrm{~mm}$. The AM1.5G solar irradiation was obtained from a $150 \mathrm{~W}$ solar simulator (SAN-EI XES-40S1) with a one-sun air mass 1.5 global filter, and a $100 \mathrm{~mW} / \mathrm{cm}^{2}$ light intensity was calibrated using a Si-based reference cell with a KG-5 filter at $25^{\circ} \mathrm{C}$. The photovoltaic parameters underwent continuous strong light soaking in a home-built test facility, consisting of a xenon lamp light source and a heater system. The solar cells were heated to $60^{\circ} \mathrm{C}$ and exposed to the xenon lamp $\left(200 \mathrm{~mW} / \mathrm{cm}^{2}\right)$ to estimate the reliability of using the recycled GaAs substrate to grow solar cells. A thermal shock test of the III-V thin-film solar cells was performed 1500 times at temperature cycles of $-40^{\circ} \mathrm{C} \sim 150^{\circ} \mathrm{C}$ at a ramp setting of $40^{\circ} \mathrm{C} / \mathrm{min}$ and a dwell time of $5 \mathrm{~min}$.

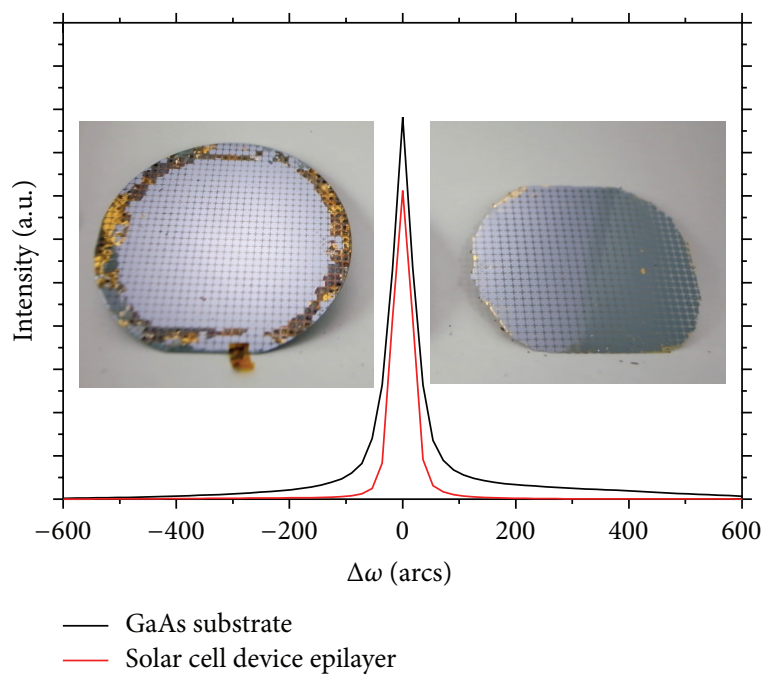

FIGURE 2: (004) XRD rocking curves of solar cell device epilayer and GaAs substrates (the $y$-axis is shown in linear scale).The left insert figure is GaAs substrate after epitaxial lift-off and without chemical cleaning; right insert figure is solar cell device epilayer transferred to the Ni substrate.

\section{Results and Discussion}

3.1. Analysis of the Recycled GaAs Substrates. Evaluating the quality of the thin epilayer transferred to the $\mathrm{Ni}$ substrate is crucial. The GaAs substrate and the solar cell device epilayer with the Ni substrate were examined by using Xray diffraction (XRD) rocking curves of the (004) reflection to confirm crystalline quality, as shown in Figure 2. Based on the XRD results, the full widths at half maximum of the $350 \mu \mathrm{m}$-thick GaAs substrate and solar cell device epilayer on the Ni substrate were 53 and 54 arcsec, respectively. The inset of Figure 2 shows a photograph of the 2 inch GaAs epilayer transferred onto the $\mathrm{Ni}$ substrate and the separated GaAs substrate before they were chemical cleaning. On the left side of Figure 2, the gold ring on the edge of the separated GaAs substrate was the residual seed layer of the electroplating process and was removed during the chemical cleaning process. The practical area of the electroplating process was the center of the blackness zone of the separated GaAs substrate. Therefore, the area of the solar cell device epilayer transferred onto the electroplated $\mathrm{Ni}$ substrate was slightly smaller than a diameter of 2 inches. The diameter of the electroplated $\mathrm{Ni}$ substrate was reduced to $6 \mathrm{~mm}$ because of the electroplating process (compared with the 2-inch GaAs substrate); the electroplated Ni substrate with solar cell device epilayer is shown on the right side of Figure 2.

Figure 3 shows the electronic properties of the single junction solar cells grown on the new and recycled GaAs substrates. The detailed performance of the initial and the 1st4 th solar cell devices is summarized in Table 1. The reversebias dark I-V characteristics in a p-n junction can serve as sensitive tools for monitoring crystalline defect reduction [8]. The solar cells can be measured to confirm the presence of recombination centers; a comparison of the solar cells 
TABLE 1: Performance of III-V solar cell grown on new/recycled GaAs substrate and GaAs thin film solar cell transferred onto Ni substrate before and after thermal shock test.

\begin{tabular}{lccccccccc}
\hline & $V_{\mathrm{oc}}(\mathrm{V})$ & $J_{\mathrm{sc}}\left(\mathrm{mA} / \mathrm{cm}^{2}\right)$ & $\mathrm{FF}(\%)$ & $\eta(\%)$ & $I_{01}(\mathrm{~A})$ & $I_{02}(\mathrm{~A})$ & $R_{s}(\Omega)$ & $R_{\text {sh }}(\Omega)$ \\
\hline & \multicolumn{7}{c}{ III-V solar cells grown on new and recycled GaAs substrate } \\
Recycled times & 0.99 & 22.59 & 81.54 & 18.23 & $1.34 \times 10^{-20}$ & $1.69 \times 10^{-13}$ & 15.6 & $3.23 \times 10^{6}$ \\
Initial device & 0.99 & 22.42 & 80.86 & 17.94 & $8.22 \times 10^{-20}$ & $2.52 \times 10^{-12}$ & 17.4 & $2.80 \times 10^{6}$ \\
1st device & 0.97 & 22.47 & 80.47 & 17.53 & $1.06 \times 10^{-19}$ & $5.22 \times 10^{-12}$ & 19.4 & $2.30 \times 10^{6}$ \\
2nd device & 0.83 & 20.64 & 76.52 & 13.10 & $2.01 \times 10^{-17}$ & $6.33 \times 10^{-11}$ & 43.5 & $1.07 \times 10^{6}$ \\
3rd device & 0.82 & 19.40 & 74.01 & 11.77 & $3.72 \times 10^{-17}$ & $4.05 \times 10^{-11}$ & 56.5 & $1.47 \times 10^{5}$ \\
4th device & &
\end{tabular}

Performance of GaAs thin films solar cell with Ni substrate before and after thermal shock test

Thermal shock test times

\begin{tabular}{lllllllll}
0 time & 0.99 & 22.37 & 80.01 & 17.71 & $3.32 \times 10^{-20}$ & $2.47 \times 10^{-12}$ & 15.8 & $3.10 \times 10^{6}$ \\
800 times & 0.98 & 21.74 & 78.48 & 16.72 & $5.54 \times 10^{-20}$ & $3.32 \times 10^{-12}$ & 21.8 & $2.10 \times 10^{6}$ \\
1200 times & 0.97 & 21.10 & 77.89 & 15.94 & $6.02 \times 10^{-20}$ & $4.24 \times 10^{-12}$ & 27.5 & $1.25 \times 10^{6}$ \\
1500 times & 0.95 & 20.19 & 76.41 & 14.65 & $1.70 \times 10^{-19}$ & $5.50 \times 10^{-12}$ & 43.3 & $2.50 \times 10^{5}$ \\
\hline
\end{tabular}

$I_{01}$ and $I_{02}$ : diode saturation current, $R_{s}$ : series resistances, and $R_{\mathrm{sh}}$ : shunt resistances.

Initial device: the GaAs solar cell grown on new GaAs substrate; 1st, 2nd, 3rd, and 4th devices: the GaAs solar cell grown on recycled GaAs substate.

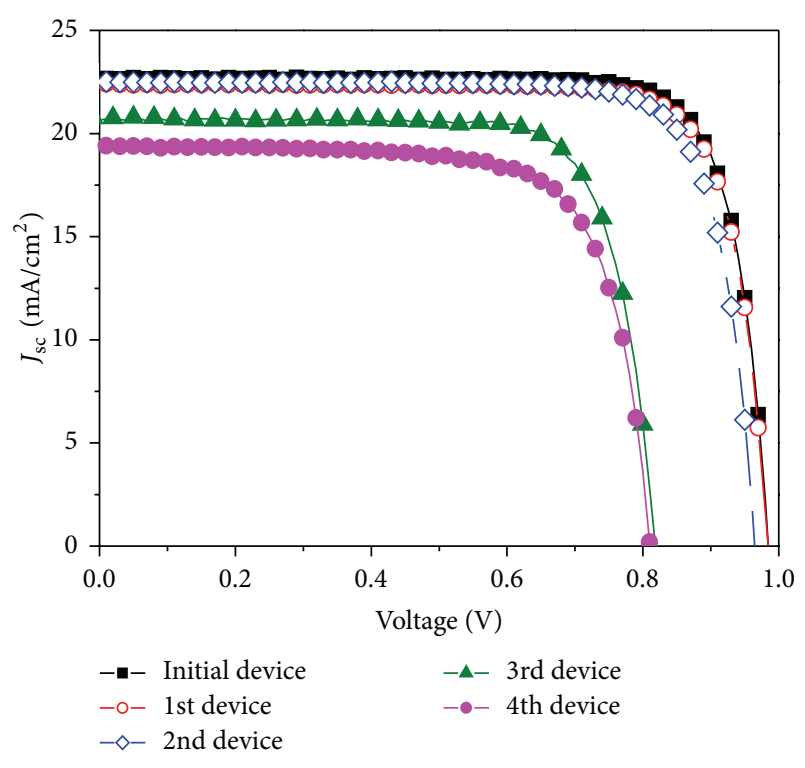

FIGURE 3: Photovoltaic performance comparison of single-junction solar cells grown on the new and recycled GaAs substrate.

grown on the new and recycled GaAs substrates is shown in Figure 4. The reverse-bias leakage current of the solar cell was increased at the reverse bias of $5 \mathrm{~V}$ when the GaAs substrate was recycled because of the high amount of recombination centers in the depletion region. The high reverse-bias leakage current was caused by defects in the solar cells; crystalline deformation and strain relaxation were expected to form recombination centers and deteriorate the performance of the solar cells by increasing the reverse-bias leakage current.

The EQE responses of the solar cells grown on the new and the recycled GaAs substrates (Figure 5) were measured to evaluate the effect of crystalline defects on photovoltaic performance. The overall profile of the EQE responses was clearly influenced by the recycled number of GaAs substrate. As shown in Figure 5(a), the EQE response of the solar cells grown on the new GaAs substrate (the initial device) was considerably higher than that of the solar cells grown on recycled GaAs substrates (the 1st-4th devices), especially at the wavelength range of 600 to $870 \mathrm{~nm}$ (details are shown in the inset of Figure 5(a)). This could be attributed to the increase in density of defects [9], such as dislocation defects, strain-relaxation induced dislocation, or recombination loss. Generally, the EQE response of a solar cell can be attributed to the following three factors: the absorption coefficient of the active layer, surface recombination velocity, and minority carrier lifetime [10]. The absorption coefficient and the surface recombination velocity are dependent on the thicknesses of the active layers and the window layer. These factors mainly depend on epilayer quality, especially minority carrier lifetime. The defective creations resulted in a short minority carrier lifetime, which contributed to the high reverse saturation current of the solar cells and the poor $\mathrm{EQE}$ responses at all wavelengths. Solar cells with higher EQE response probably have longer minority-carrier lifetimes. As shown in Figure 5(b), the EQE responses of the solar cells grown on the new and the recycled GaAs substrates measured after a light soaking test were lower than those measured before the light soaking test.

3.2. Reliability Analysis of the III-V Thin-Film Solar Cell. The thermal shock test (TST) is crucial for III-V thin-film solar cells with Ni substrates because of the considerable mismatch in thermal expansion coefficients between the epilayers $(6.5 \times$ $\left.10^{-6} / \mathrm{K}\right)$ and $\mathrm{Ni}\left(13.4 \times 10^{-6} / \mathrm{K}\right)$. The performance of the thinfilm GaAs solar cells after conducting 1500 thermal shock tests is shown in Figure 6. The performance of 20 cell samples was summarized, with an error bar to further evaluate the quality of the thin-film GaAs solar cells. The 20 different cell samples were selected from two-time experimental results. The corresponding open circuit voltage $\left(V_{\text {oc }}\right)$, short circuit 


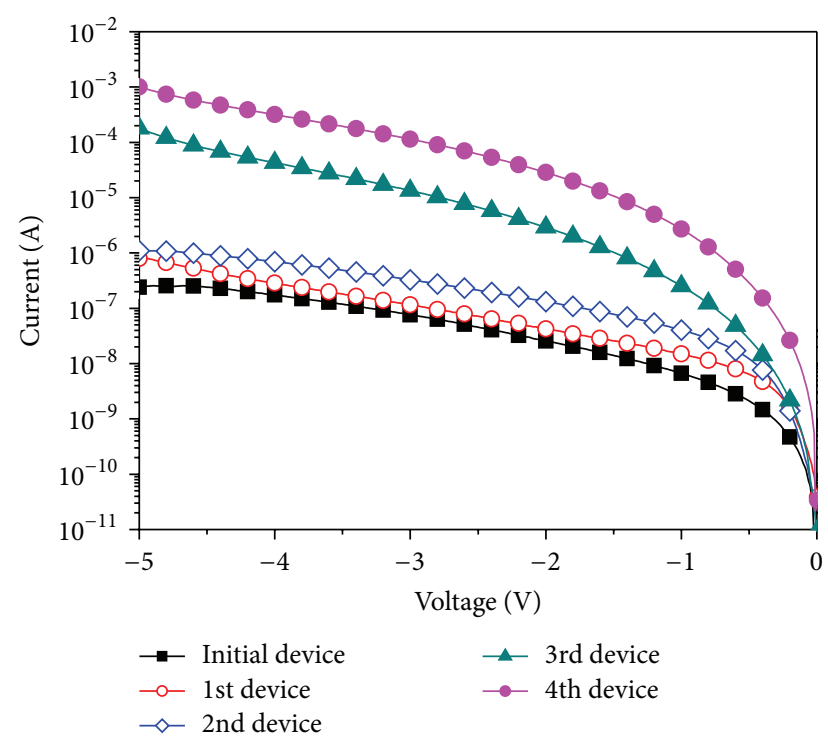

FIGURE 4: Reverse-bias dark I-V characteristics of the solar cells grown on new and recycled GaAs substrate.

current density $\left(J_{\mathrm{sc}}\right)$, fill factor $(\mathrm{FF})$, and $\eta$ for the TF cells were collected from the closed square point of the $y$-axis by using an error bar to further investigate the degradation of thin-film solar cells (summarized in Table 1).

The performance of the TF-cells did not substantially degrade before 800 thermal shock tests. After 1200 thermal shock tests, the performance of the TF-cells was $90 \%$ lower than the initial performance. The degradation of TFcells could be attributed to two reasons: the presence of a microcrack in the epilayer or microvoids in the electroplated substrate/seed layer interface or seed layer/epilayer interface. Microcracks can cause a solar cell's current leakage rate to increase, which deteriorates $V_{\text {oc }}$ and $J_{\text {sc }}$ [11]. Moreover, the microvoids could cause the deformation of the GaAs epilayer, resulting in dislocation (misfit or threading dislocations) at the heteroepitaxial interface. The distortion of the heteroepitaxial interface causes the doping concentration of the epilayer to decrease, which further reduces the $\mathrm{FF}[12,13]$.

A scanning acoustic microscope (SAM) equipped with a $140 \mathrm{MHz}$ probe (SAM, Hitachi FS300II) was used to examine whether microcracks or microvoids existed in the the electroplated substrate/seed layer or seed layer/epilayer interfaces after the thermal shock tests. The SAM images (shown in Figure 7) show that no substantial microcracks existed in the epilayer with the Ni substrate.

Moreover, scanning electron microscopy (SEM) was performed to examine the surface morphology of the TF-cells after the thermal shock tests were conducted and to further observe the microcracks or microvoids. Figure 8(a) shows the electrode pattern for the TF cells. Figures $8(b)-8(d)$ show the surface morphologies of the TF cells at amplification factors of $3000 \times, 5000 \times$, and $7000 \times$. The SEM images did not show substantial microcracks in the epilayer, on which the thermal shock test was performed 1500 times. Two reasons could explain this observation. First, SAM and, especially, SEM cannot provide overall observations of high amplification factors. The observational image area was small for high amplification factors; therefore, some details could have been missed when performing SEM. Second, the deformation of the GaAs epilayer may have caused dislocation (misfit or threading dislocations) in the epilayer structure. The dislocation could not propagate to the surface; therefore, it could not be observed on the surface by using SEM or SAM. The SEM and SAM images cannot exhibit distortion of the crystalline structure or dislocation (misfit or threading dislocations) at the epilayer structure, confirming the reason for the deterioration of the GaAs thin-film solar cells transferred onto the electroplated substrate after thermal shock tests. The reverse-bias dark I-V curves and double diode modes of the solar cells are provided in Section C to further understand the degradation of thin-film solar cells.

The EQE responses of the solar cells transferred onto the electroplated $\mathrm{Ni}$ substrate undergone various numbers of thermal shock test times, which were used to evaluate the effects of mismatched thermal expansion coefficients on the photovoltaic performance, are shown in Figure 9. The overall profiles of the EQE responses were clearly influenced by the number of thermal shock tests. This could be because of the substantial mismatch in thermal expansion coefficients that distorted the crystalline quality of the active layers by generating dislocations (e.g., the increased leakage current and recombination loss). The dislocations were associated with threading dislocations, which propagate upward through the epilayer to the surface or active layer of solar cells. The propagation of dislocations influences the interface defects of solar cells in the heterostructure (e.g., the window/emitter layer and base/BSF layer). The performance of solar cells is considerably influenced by the heterostructure design.

Two types of surface recombination occurred in the solar cells. The first type of surface recombination (labeled $S_{p}$ ) occurred in the interface of the windows/emitter layer, and the second type (labeled $S_{n}$ ) occurred in the interface of the base/BSF layer. A high $S_{p}$ caused the blue response (the range of short wavelengths) $[14,15]$ to decrease dramatically. However, a high $S_{p}$ also caused a reduction in the red response (the range of long wavelengths). By contrast, a high $S_{n}$ caused a reduction only the in red response [16]. An increase in the surface recombination of solar cells also increases the dark current of the solar cells, reducing the $V_{\mathrm{oc}}$. To confirm this observation, the double diode mode of the solar cells and the reverse-bias dark I-V characteristics are provided.

The reverse-bias leakage currents of the solar cells were measured to confirm the presence of recombination centers. The comparisons of solar cells that underwent various numbers of thermal shock tests are shown in Figure 10. When 800 thermal shock tests were performed, the reverse-bias leakage currents of solar cells were drastically increased at the reverse bias of $5 \mathrm{~V}$ because of the great amount of recombination centers in the depletion region. A dramatic decrease of 3 


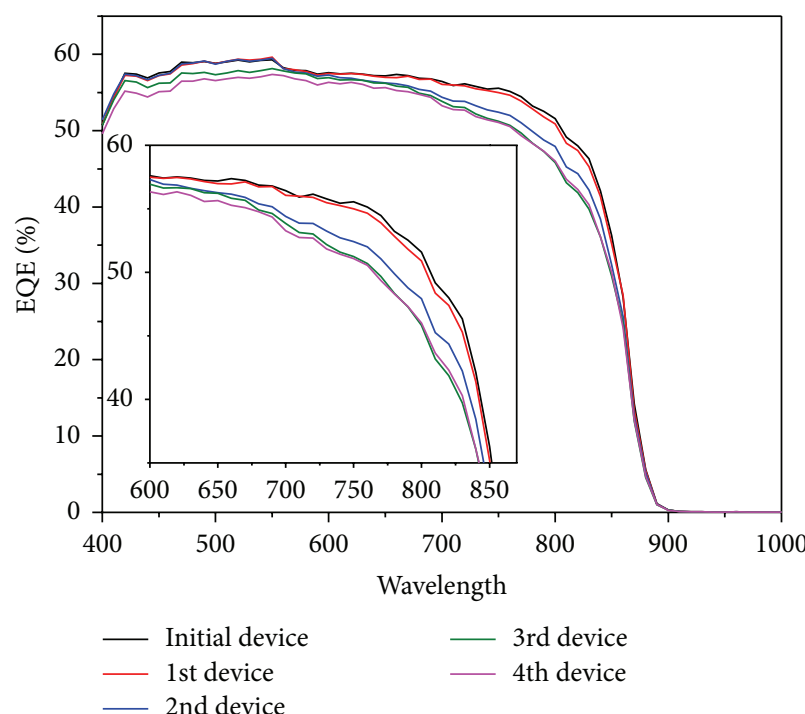

(a)

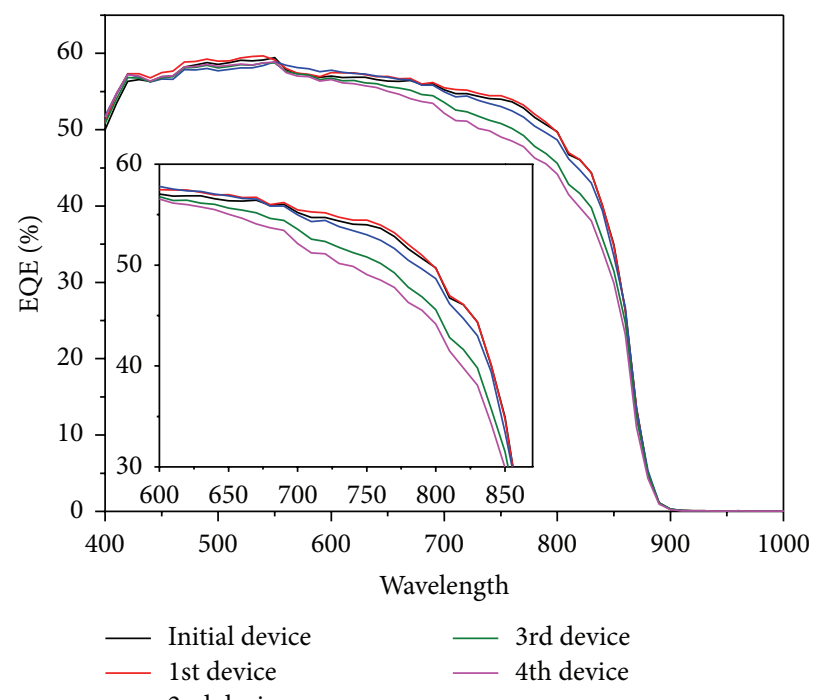

(b)

FIGURE 5: EQE responses of solar cell grown on new and recycled GaAs substrate; (a) before light soaking test (b) with light soaking test of 267 hours. (The inset figure is the magnification of EQE curve at wavelength from 600 to $870 \mathrm{~nm}$.)

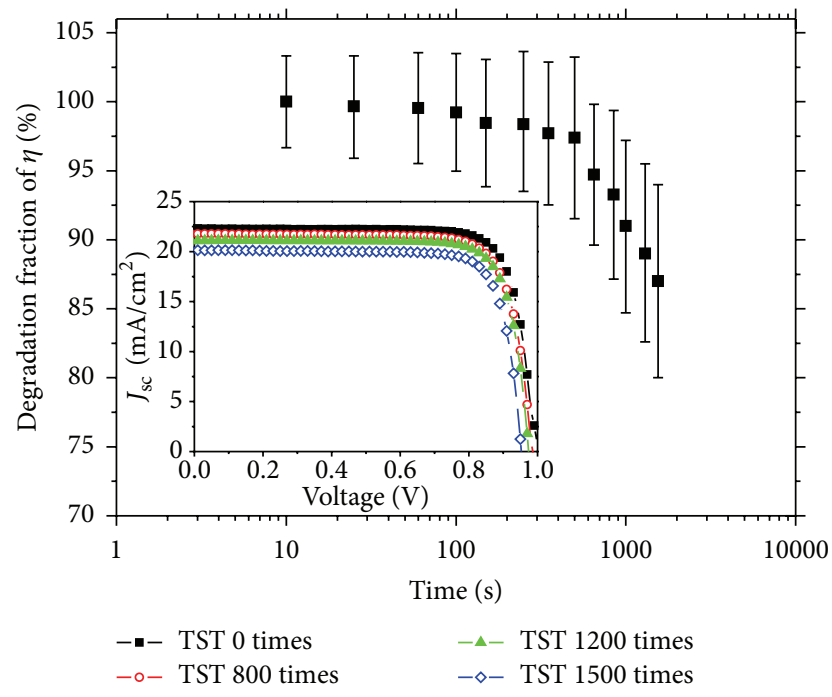

FIGURE 6: The degradation faction of conversion efficiency for GaAs thin-film solar cell transferred to electroplated Ni substrate after thermal shock test (cycle time are $0,800,1200$, and 1500 times). The insert is the I-V curve of thin-films GaAs solar cell with different thermal shock test times.

orders of magnitude in the reverse-bias leakage currents of solar cells from 0 to 1500 thermal shock tests was at the reverse bias of $5 \mathrm{~V}$ because of the dislocation generated from microcracks or microvoids.

3.3. Analysis of the Double-Diode Model of Solar Cells. The double-diode model of solar cells under illumination conditions was used to fit the I-V curve using (1) [17]. The solar cells

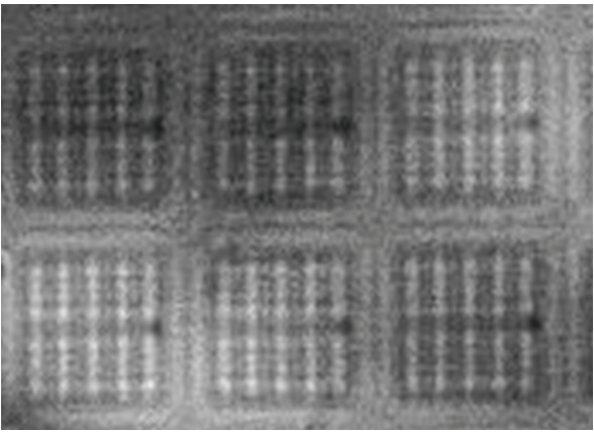

FIGURE 7: SAM images of TF-cells after the thermal shock test.

grown on the new and the recycled GaAs substrates and the GaAs solar cell transferred onto the electroplated Ni substrate as thin-film solar cell which underwent various numbers of thermal shock tests, are given as follows:

$$
\begin{aligned}
I= & I_{p h}-I_{01}\left(\exp \frac{q\left(V+I R_{s}\right)}{k T}-1\right) \\
& -I_{02}\left(\exp \frac{V+I R_{s}}{2 k T}-1\right)-\frac{V+I R_{s}}{R_{\mathrm{sh}}},
\end{aligned}
$$

where $k$ is the Boltzmann's constant, $T$ is the cell's temperature, $q$ is the elementary charge, $I_{\mathrm{ph}}$ is the photocurrent, $I_{01}$ and $I_{02}$ are the diode saturation currents (with diode ideality factors of 1 and 2, resp.), and $R_{s}$ and $R_{\mathrm{sh}}$ are the series resistance and shunt resistance, respectively. The values of $I_{01}$ can be associated with similar minority carrier diffusion lengths and interface recombination velocities. The $I_{02}$ corresponds with the recombination in the depletion 


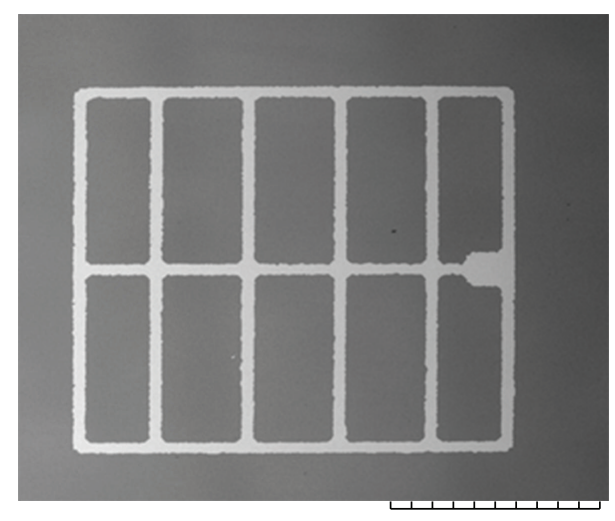

TM-1000_2533

L $\quad$ D2.6 $\times 120 \quad 500 \mu \mathrm{m}$

(a)

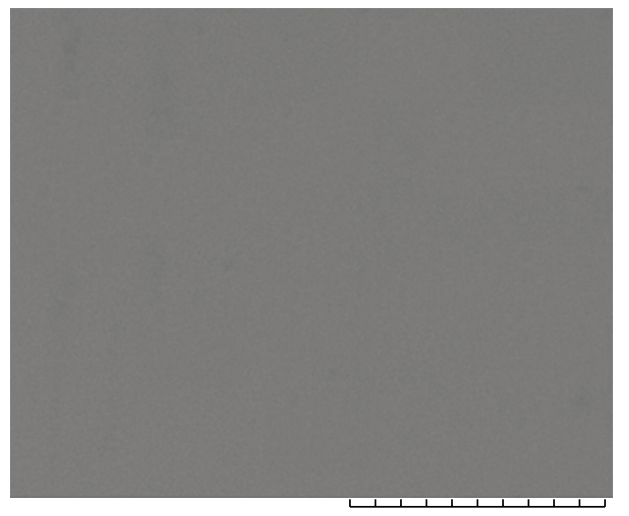

TM-1000_2536
L D $2.6 \times 5.0 \mathrm{k} 20 \mu \mathrm{m}$

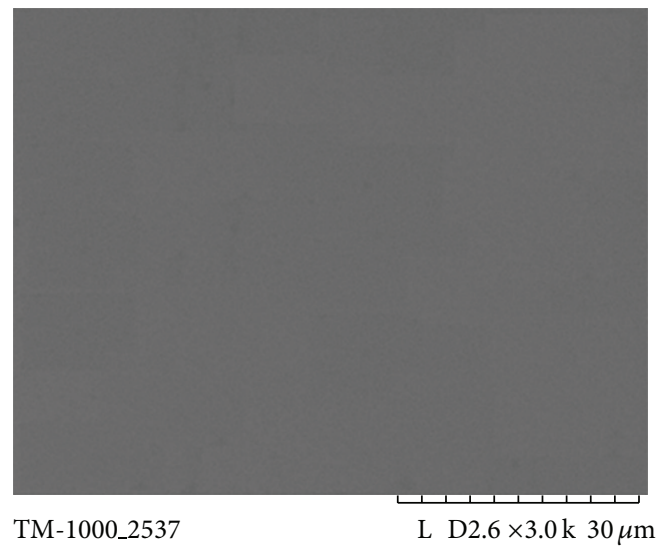

(b)

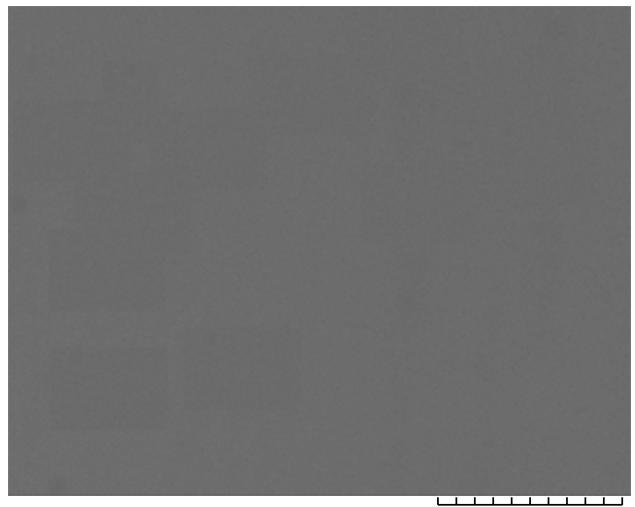

TM-1000_2535

L D2.6 $\times 7.0 \mathrm{k} 10 \mu \mathrm{m}$

(c)

(d)

FIGURE 8: SEM images of the TF cell after the thermal shock test; (a) electrode pattern for the TF-cell; surface morphology images of the TF cell with amplification factors of (b) $3000 \times$, (c) $5000 \times$, and (d) $7000 \times$.

region. If the number of crystalline defects increases substantially, the recombination of minority carriers occurs in the depletion region, which, in turn, contributes to items in the $I_{02}[18]$.

The performance of solar cells grown on new and recycled GaAs substrates and GaAs thin-film solar cells grown on electroplated Ni substrates, which underwent various numbers of thermal shock tests, are summarized in Table 1. For the recycled GaAs substrates, the $V_{\text {oc }}$ and FF of the solar cells grown on new or recycled GaAs substrates were considerably deteriorated. The $V_{\text {oc }}$ of solar cells grown on recycled GaAs substrates decreased when recycled more than 2 times. A low $V_{\text {oc }}$ could be caused by solar cell interface defects in the heterostructure because the surface roughness of the recycled GaAs substrate rapidly increased from $0.255 \mathrm{~nm}$ to $4.420 \mathrm{~nm}$. It was reported that a low $V_{\text {oc }}$ results from high diode saturation currents $\left(I_{0}\right)$ in solar cells. A high diode saturation current was attributable to the recombination loss caused by defects in both the quasineutral and space-charge regions of the solar cell $[19,20]$.

The $I_{01}$ and $I_{02}$ values of the solar cells grown on the new and the recycled GaAs substrates were considerably altered by the number of recycled times. A dramatic decrease of 3 and 2 orders of magnitude in the $I_{01}$ and $I_{02}$ terms from the initial device to the 4 th ELO TF cell was observed because of the uneven surface morphology, especially for the $I_{01}$ term.

The $I_{02}$ increased because of two factors. The first factor was the size of the high concentrator solar cells. They are inherently small devices that have huge perimeter-toarea ratios; therefore, they are strongly affected by changes in the recombination properties of the perimeter (a main contribution to the $I_{02}$ factor is the recombination at the PN junction [18]). Another possible factor was the generation of a great number of dislocations in the space charge region [20]. If the amount of dislocations considerably increases, then the recombination in the space charge regions contributes to $I_{02}$.

The reduction in the FF is principally attributable to two factors. The first factor is the high series resistance $\left(R_{s}\right)$ and shunt resistance $\left(R_{\mathrm{sh}}\right)$; the second factor is the low doping level of the emitter layer to increase $R_{s}$. The principal factor in most studies has been $R_{s}$. The FF considerably decayed in the 3rd and 4th ELO TF-cells compared with that of the initial cell. In this study, the low FF of the 3rd and 4th ELO TF-cells is attributable to poor crystalline quality and the deteriorated doping-level of the contact layer caused by 


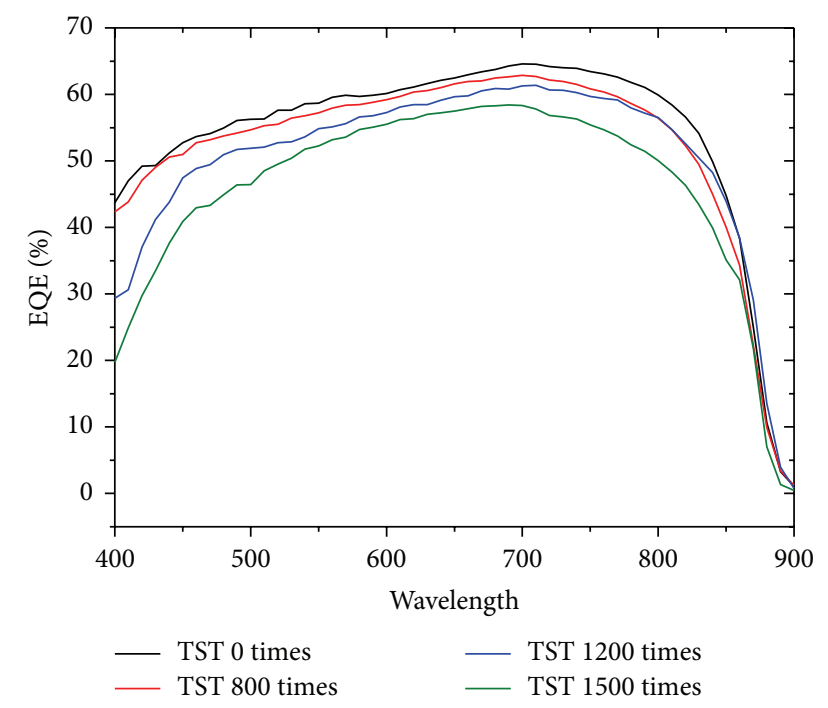

FIGURE 9: EQE responses of thin-film solar cell grown on electroplated Ni substrate with different thermal shock times.

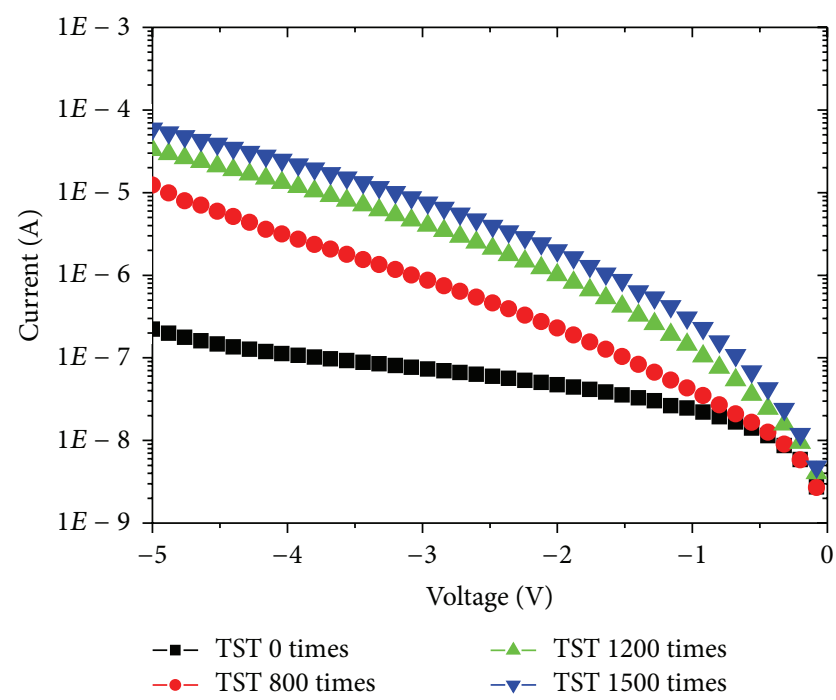

Figure 10: Reverse-bias dark I-V characteristics of the thin-film solar cells grown on electroplate nickel substrate.

the high surface roughness of the recycled GaAs substrate or wafer contamination.

For the GaAs thin-film solar cells grown on the electroplated Ni substrate, a dramatic decrease in 1 order of magnitude in the $I_{01}$ term from 0 to 1500 thermal shock tests was observed because of microcracks or microvoids in the active layer or interface of the heterojunction. The microcracks or microvoids were generated during thermal shock tests because of a great mismatch in thermal expansion coefficients. The variation range of the $I_{02}$ term did not considerably exceed 1 order of magnitude. Because of the high crystalline quality of the active layer, the different thermal shock tests did not considerably deteriorate the TF-cell.

\section{Conclusion}

A reliability analysis of III-V solar cells grown on new and recycled GaAs substrates and a III-V solar cell transferred onto an electroplated Ni substrate as III-V thin-film solar cell are reported. For the solar cells grown on the new and the recycled GaAs substrates, the main factor for the degradation of solar cell performance was the surface morphology of the recycled GaAs substrate. The uneven surface morphology caused the quality of the subsequently active layer of the solar cell to deteriorate and affected the $V_{\mathrm{oc}}$ and FF. The performance of the TF cell that underwent numerous shock tests was not considerably influenced after 800 thermal shock tests. After 1500 thermal shock tests, a substantial amount of microcracks or microvoids were generated in the active layer of the solar cell, which deteriorated the performance, especially the EQE response of the solar cell.

\section{Acknowledgment}

This work was financially supported by the National Science Council of the Republic of China, under the contract numbers NSC 101-2218-E-451-002 and NSC 99-2221-E-006-097MY3.

\section{References}

[1] J. F. Geisz, D. J. Friedman, J. S. Ward et al., “40.8\% efficient inverted triple-junction solar cell with two independently metamorphic junctions," Applied Physics Letters, vol. 93, no. 12, Article ID 123505, 2008.

[2] J. F. Geisz, S. Kurtz, M. W. Wanlass et al., "High-efficiency GaInP/GaAs/InGaAs triple-junction solar cells grown inverted with a metamorphic bottom junction," Applied Physics Letters, vol. 91, no. 2, Article ID 023502, 2007.

[3] D. C. Law, K. M. Edmondson, N. Siddiqi et al., "Lightweight, flexible, high-efficiency III-V multijunction cells," in Proceedings of the Conference Record of the IEEE 4th World Conference on Photovoltaic Energy Conversion (WCPEC-4 '06), pp. 18791882, Waikoloa, Hawaii, USA, May 2006.

[4] M.-C. Tseng, R.-H. Horng, F.-L. Wu, C.-H. Wu, and M.D. Yang, "Performance of GaAs/mirror/Cu-substrate thin-film solar cells," IEEE Transactions on Electron Devices, vol. 58, no. 11, pp. 3898-3904, 2011.

[5] C. W. Cheng, K. T. Shiu, N. Li, S. J. Han, L. Shi, and D. K. Sadana, "Epitaxial lift-off process for gallium arsenide substrate reuse and flexible electronics," Nature Communications, vol. 4, pp. 1-4, 2013.

[6] G. J. Bauhuis, P. Mulder, E. J. Haverkamp et al., "Wafer reuse for repeated growth of III-V solar cells," Progress in Photovoltaics, vol. 18, no. 3, pp. 155-159, 2010.

[7] R.-H. Horng, M.-C. Tseng, F.-L. Wu, C.-H. Li, C.-H. Wu, and M.-D. Yang, "Thin film solar cells fabricated using cross-shaped pattern epilayer lift-off technology for substrate recycling applications," IEEE Transactions on Electron Devices, vol. 59, no. 3, pp. 666-672, 2012.

[8] P. Kozodoy, J. P. Ibbetson, H. Marchand et al., "Electrical characterization of GaN p-n junctions with and without threading dislocations," Applied Physics Letters, vol. 73, no. 7, pp. 975-977, 1998. 
[9] T. Sasaki, K. Arafune, H. S. Lee et al., "Effects of thermal cycle annealing on reduction of defect density in lattice-mismatched InGaAs solar cells," Physica B, vol. 376-377, no. 1, pp. 626-629, 2006.

[10] J. Nelson, The Physics of Solar Cells, Imperial College Press, London, UK, 2003.

[11] I. Rey-Stolle and C. Algora, "Analysis of wirebonding techniques for contacting high concentrator solar cells," IEEE Transactions on Advanced Packaging, vol. 26, no. 1, pp. 47-53, 2003.

[12] T. Takamoto, M. Kaneiwa, M. Imaizumi, and M. Yamaguchi, "InGaP/GaAs-based multijunction solar cells," Progress in Photovoltaics, vol. 13, no. 6, pp. 495-511, 2005.

[13] T. Takamoto, T. Kodama, H. Yamaguchi et al., "Paper-thin InGaP/GaAs solar cells," in Proceedings of the Conference Record of the IEEE 4th World Conference on Photovoltaic Energy Conversion (WCPEC-4 '06), pp. 1769-1772, Waikoloa, Hawaii, USA, May 2006.

[14] R. Jaakkola, J. Lammasniemi, A. B. Kazantsev, and K. Tappura, "Comparison of $\mathrm{Al}_{0.51} \mathrm{In}_{0.49} \mathrm{P}$ and $\mathrm{Ga}_{0.51} \mathrm{In}_{0.49} \mathrm{P}$ window layers for GaAs and GaInAsP solar cells," in Proceedings of the IEEE 26th Photovoltaic Specialists Conference, pp. 891-894, October 1997.

[15] G. Létay, M. Hermle, and A. W. Bett, "Simulating singlejunction GaAs solar cells including photon recycling," Progress in Photovoltaics, vol. 14, no. 8, pp. 683-696, 2006.

[16] B. Galiana, I. Rey-Stolle, M. Baudrit, I. García, and C. Algora, "A comparative study of BSF layers for GaAs-based single-junction or multijunction concentrator solar cells," Semiconductor Science and Technology, vol. 21, no. 10, article no. 003, pp. 1387-1392, 2006.

[17] D. S. H. Chan and J. C. H. Phang, "Analytical methods for the extraction of solar-cell single- and double-diode model parameters from I-V characteristics," IEEE Transactions on Electron Devices, vol. 34, no. 2, pp. 286-293, 1987.

[18] V. diaz and C. Algora, "The influence of perimeter recombination in the design of very high concentrator GaAs solar cells," in Proceedings of the of 16th European PVSEC, pp. 1034-1037, Glasgow, Scotland, 2000.

[19] A. Luque and S. Hegedus, Handbook of Photovoltaic Science and Engineering, Wiley, Hoboken, NJ, USA, 2003.

[20] J. C. Zolper and A. M. Barnett, "The Effect of dislocations on the open-circuit voltage of gallium arsenide solar cells," IEEE Transactions on Electron Devices, vol. 37, no. 2, pp. 478-484, 1990. 

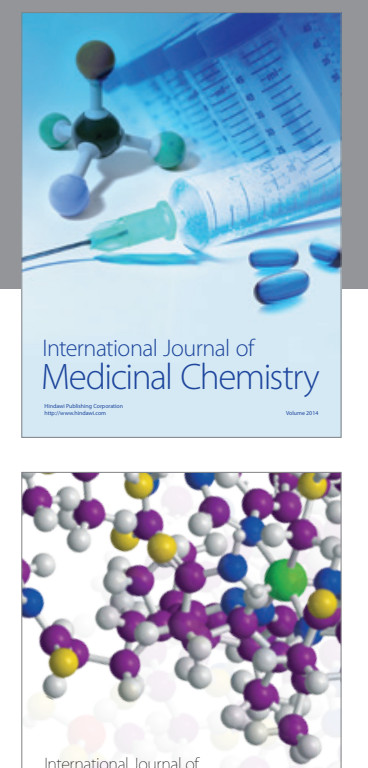

\section{Carbohydrate} Chemistry

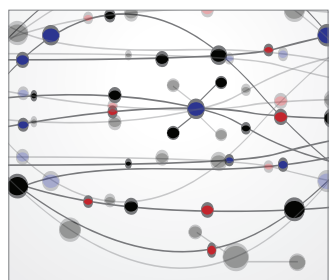

The Scientific World Journal
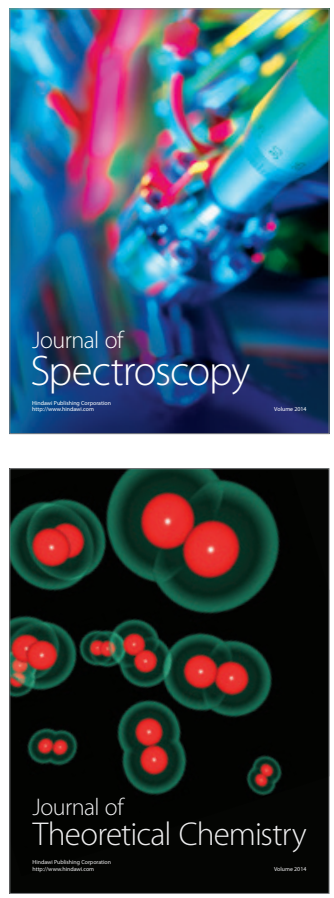
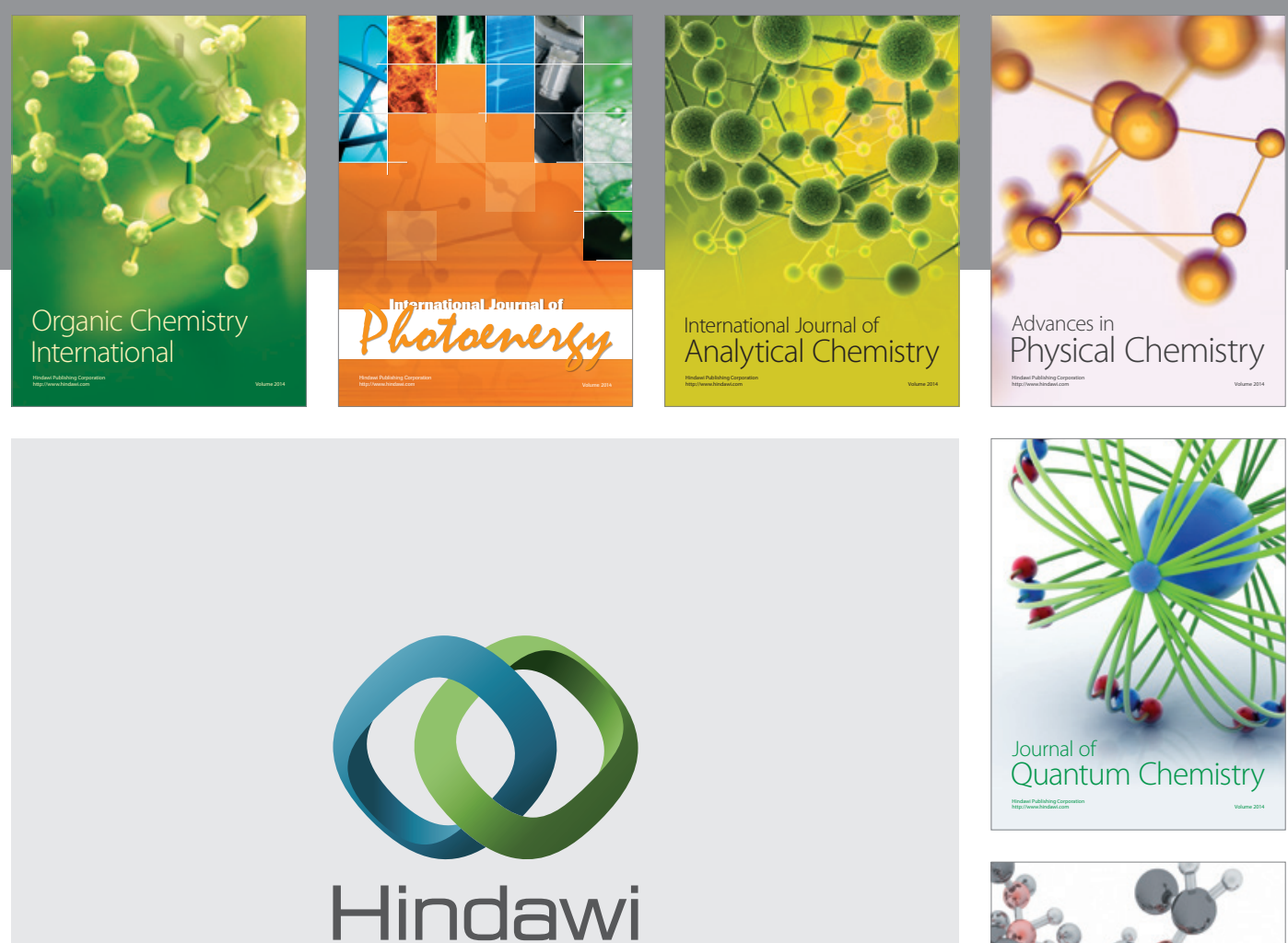

Submit your manuscripts at

http://www.hindawi.com

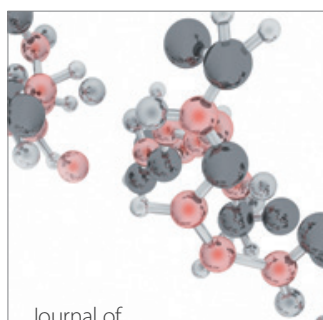

Analytical Methods

in Chemistry

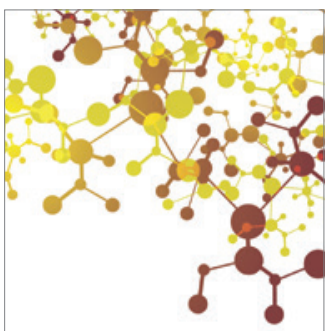

Journal of

Applied Chemistry

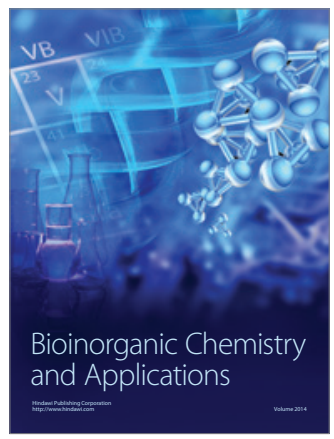

Inorganic Chemistry
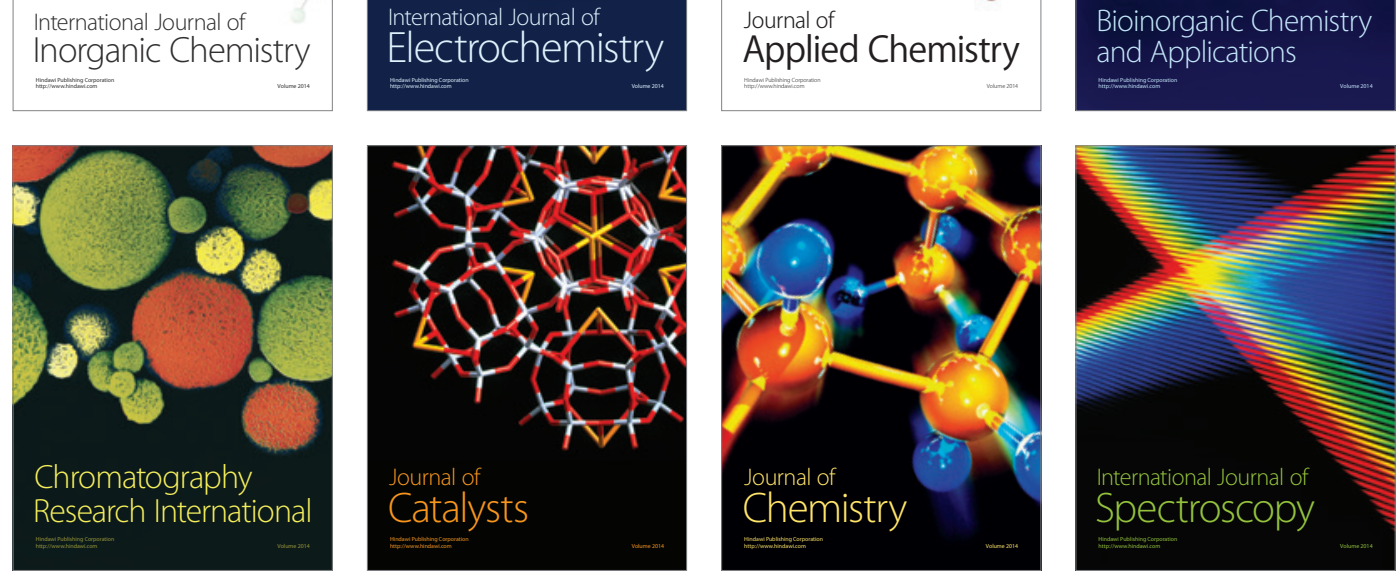